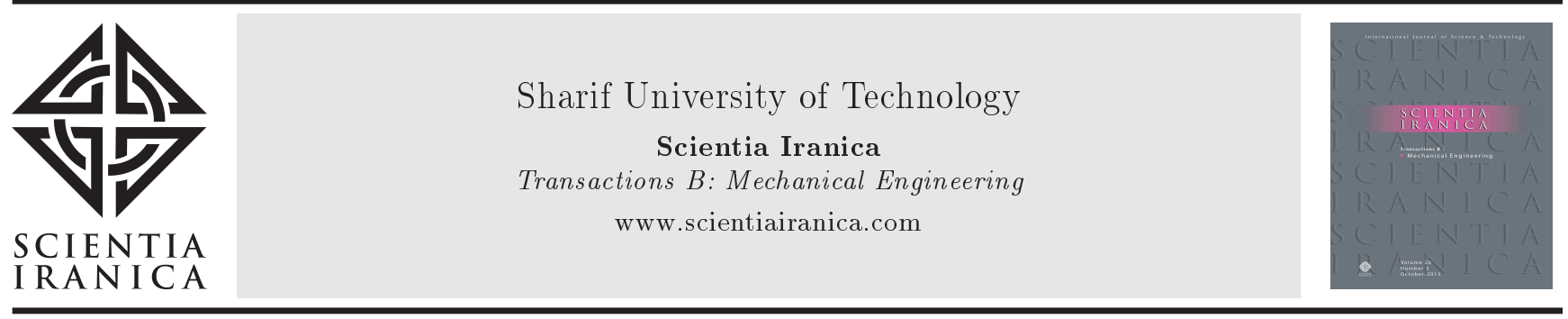

\title{
A novel computational framework to approximate the analytical solution of a nonlinear fractional elastic beam equation
}

\author{
K. Sayevand* and K. Pichaghchi \\ Faculty of Mathematical Sciences, University of Malayer, Malayer, P.O. Box 16846-13114, Iran.
}

Received 15 November 2014; received in revised form 9 February 2015; accepted 4 May 2015

KEYWORDS
Caputo fractional
derivative;
Nonlinear elastic
beam equation;
Fourier transform;
Laplace transform;
Homotopy
perturbation method.

\section{KEYWORDS}

derivative;

Nonlinear elastic

beam equation;

perturbation method.

\begin{abstract}
In this paper, the generalized travelling solutions of the nonlinear fractional beam equation is investigated by means of the homotopy perturbation method. The fractional derivative is described in the Caputo sense. The reliability and potential of the proposed approach, which is based on joint Fourier-Laplace transforms and the homotopy perturbation method, will be discussed. The solutions can be approximated via an analytical series solution. Moreover, the convergence and stability of the proposed approach for this equation is investigated, and the results reveal that the proposed scheme is very effective and promising.
\end{abstract}

(C) 2016 Sharif University of Technology. All rights reserved.

\section{Introduction}

Many partial differential equations are widely used to describe important phenomena in mathematics, physics and engineering [1,2]. In recent years, it has been discovered that differential equations involving derivatives of a non-integer order can be adequate models for various physical phenomena [3-10]. The present paper is a study on developing a fairly complete theoretical understanding of the solutions for beam equations with fractional coordinate derivatives. In the nonlinear elastic beam theory, the stress in the lateral direction is neglected, and governing equations can be considered in the following form, when $\xi$ is the deflection of the beam, and $a$ and $b$ are parameters:

$$
\frac{\partial^{\eta} \xi(x, t)}{\partial t^{\eta}}+\frac{\partial^{\gamma} \xi(x, t)}{\partial x^{\gamma}}+a \frac{\partial^{\delta} \xi(x, t)}{\partial x^{\delta}}+b \xi(x, t)+\xi^{n}(x, t)=0,
$$

*. Corresponding author. Tel./Fax: +98 8133340867 E-mail addresses: ksayehvand@malayeru.ac.ir (K. Sayevand); kazem.pichaghchi@malayeru.ac.ir. (K. Pichaghchi) where:

$$
\begin{aligned}
& 0<\eta, \quad \delta \leq 2, \quad 0<\gamma \leq 4, \\
& a, b \in \mathbb{R}, \quad n \in \mathbb{N} .
\end{aligned}
$$

This problem has many applications in engineering systems. Some of these applications are vibrations that occur in bridges and rail-road tracks due to moving vehicles, vibrations that occur in pipe systems due to fluid flow, machining operations, machine chains and belt drives, and thermal processing subjected to moving heat sources.

In this paper, we aim to establish application of the homotopy perturbation method to derive solutions for mathematical variants of the fractional beam equation. The homotopy perturbation method [4,5,11-13] was first proposed by Prof. He in order to solve various nonlinear problems [11]. This promising analytic technique has successfully been applied to solving many types of linear and nonlinear functional equations. The homotopy perturbation method, in contrast to the traditional perturbation method, does not require a small parameter and is constructed with an embedding 
parameter, $p \in[0,1]$, which is considered a small parameter. Considerable research work has recently been conducted in application of this method to fractional advection-dispersion equations, multi-order fractional differential equations, Navier-Stokes equations, nonlinear Schrödinger equations, Volterra integro-differential equations, nonlinear oscillators, boundary value problems, fractional KdV equations, quadratic Riccati differential equations of fractional order and many others. For more details about the homotopy perturbation method and its applications, the reader is advised to consult the results of research work presented in [1419]. All these successful applications verified the effectiveness, flexibility, and validity of the homotopy perturbation method.

The paper is organized as follows. After a short background on fractional calculus and mathematical prerequisites, the nonlinear fractional beam equation is investigated by means of joint Fourier-Laplace transforms and the homotopy perturbation method. A short summary with discussion is given in the final section.

\section{Mathematical prerequisites}

Definitions of the well-known Fourier and Laplace transforms of function $\xi(x, t)$ and their inverses are described below [20]:

Definition 1. The Laplace transform of function $\xi(x$, $t$ ) (continuous or partially continuous and of exponential order as $t$ approaches infinity), with respect to $t$, is defined by:

$$
\begin{aligned}
& \mathcal{L}\{\xi(x, t)\}=\tilde{\xi}(x, s)=\int_{0}^{\infty} e^{-s t} \xi(x, t) d t, \\
& (t>0, \quad x \in \mathbb{R}),
\end{aligned}
$$

where $\mathfrak{R e}(s)>0$, and its inverse transform, with respect to $s$, is given by:

$$
\mathcal{L}^{-1}\{\tilde{\xi}(x, s)\}=\xi(x, t)=\frac{1}{2 \pi i} \int_{\theta-i \infty}^{\theta+i \infty} e^{s t} \xi(x, s) d s
$$

where $\theta$ is a fixed real number.

Definition 2. The Fourier transform of function $\xi(x$, $t)$, with respect to $x$, is defined by:

$$
\mathcal{F}\{\xi(x, t)\}=\hat{\xi}(\omega, t)=\int_{-\infty}^{\infty} e^{i \omega x} \xi(x, t) d x, \quad(\omega>0)
$$

and the inverse Fourier transform with respect to $\omega$, is given by the formula:

$$
\mathcal{F}^{-1}\{\hat{\xi}(\omega, t)\}=\xi(x, t)=\frac{1}{2 \pi} \int_{-\infty}^{\infty} e^{-i \omega x} \hat{\xi}(\omega, t) d \omega
$$

Definition 3. The Mittag-Leffler function, $E_{\alpha, \beta}(z)$ with $\alpha>0, \beta>0$, is defined by the following series representation, valid in the whole complex plane:

$$
E_{\alpha, \beta}(z)=\sum_{n=0}^{\infty} \frac{z^{n}}{\Gamma(n \alpha+\beta)}, \quad z \in \mathbb{C} .
$$

For $\beta=1$, we obtain the Mittag-Leffler function in one parameter:

$$
E_{\alpha, 1}(z)=\sum_{n=0}^{\infty} \frac{z^{n}}{\Gamma(n \alpha+1)} \equiv E_{\alpha}(z) .
$$

Definition 4. The general $H$-function is defined as the inverse Mellin transform [21]:

$$
\begin{aligned}
& H_{P, Q}^{m, n}\left(\left.z\right|_{\left(b_{1}, B_{1}\right) \cdots\left(b_{Q}, B_{Q}\right)} ^{\left(a_{1}, A_{1}\right) \cdots\left(a_{P}, A_{p}\right)}\right) \\
& =\frac{1}{2 \pi i} \int_{C} \frac{\prod_{j=1}^{m} \Gamma\left(b_{j}-B_{j} s\right) \prod_{j=1}^{n} \Gamma\left(1-a_{j}+A_{j} s\right)}{\prod_{j=m+1}^{Q} \Gamma\left(1-b_{j}+B_{j} s\right) \prod_{j=n+1}^{P} \Gamma\left(a_{j}-A_{j} s\right)} z^{s} d s
\end{aligned}
$$

where contour $C$ runs from $C-i \infty$ to $C+i \infty$, separating the poles of $\Gamma\left(b_{j}-B_{j} s\right),(j=1, \cdots, m)$, from those of $\Gamma\left(1-a_{j}+A_{j} s\right),(j=1, \cdots, n)$. The integers, $m, n, P$ and $Q$, satisfy $0 \leq m \leq Q$ and $0 \leq n \leq P$. The coefficients, $A_{j}$ and $B_{j}$ are positive real numbers and the complex parameters, $a_{j}$ and $b_{j}$ are such that no poles in the integrand coincide. If:

$$
\Omega=\sum_{j=1}^{n} A_{j}-\sum_{j=n+1}^{P} A_{j}+\sum_{j=1}^{m} B_{j}-\sum_{j=m+1}^{Q} B_{j}>0
$$

then, the integral converges absolutely and defines the $H$-function in the sector $|\arg z|<\Omega \frac{\pi}{2}$.

\subsection{Background on fractional derivatives}

There are several definitions for fractional differential equations. These definitions include GrunwaldLetnikov, Riemann-Liouville, Caputo, Weyl, Marchaud, Riesz, and Jumarie fractional derivatives. Here, some preliminaries and notations regarding fractional calculus are presented.

Definition 5. The Caputo fractional derivative of order $\alpha$ is defined as:

$$
D_{* t}^{\alpha} \xi(t)=\left\{\begin{array}{c}
\frac{1}{\Gamma(m-\alpha)} \int_{0}^{t}(t-\tau)^{m-\alpha-1} \xi^{(m)}(\tau) d \tau, \\
\alpha>0, \quad t>0, \\
m-1<\alpha<m, \quad m \in \mathbb{N}, \\
\frac{d^{m}}{d t^{m}} \xi(t), \quad \alpha=m,
\end{array}\right.
$$

where parameter $\alpha$ is the order of the derivative and 
is allowed to be real or even complex. In this paper, only real and positive $\alpha$ will be considered. With this definition, a fractional derivative would be defined for a differentiable function only [22]. Recently, to overcome this limitation and in order to deal with nondifferentiable functions, the following definitions are presented.

Definition 6. We can define a fractional derivative in the form [23]:

$D_{t}^{\alpha} \xi(t)=\frac{1}{\Gamma(m-\alpha)} \frac{d^{m}}{d t^{m}} \int_{t_{0}}^{t}(\tau-t)^{m-\alpha-1}\left(\xi_{0}(\tau)-\xi(\tau)\right) d \tau$.

For a continuous and differentiable, $\xi$, we have:

$$
\begin{aligned}
\xi_{0}(t)= & \xi\left(t_{0}\right)+\left(t-t_{0}\right) \xi^{\prime}\left(t_{0}\right)+\frac{1}{2}\left(t-t_{0}\right)^{2} \xi^{\prime \prime}\left(t_{0}\right) \\
& +\cdots+\frac{1}{(m-1) !}\left(t-t_{0}\right)^{n-1} \xi^{(m-1)}\left(t_{0}\right) .
\end{aligned}
$$

If $\xi_{0}(t)$ is continuous but not differentiable anywhere, we have:

$$
\begin{aligned}
\xi_{0}(t)= & \xi\left(t_{0}\right)+\frac{\left(t-t_{0}\right)}{\Gamma(1+\alpha)} \xi^{(\alpha)}\left(t_{0}\right)+\frac{\left(t-t_{0}\right)^{2}}{\Gamma(1+2 \alpha)} \xi^{(2 \alpha)}\left(t_{0}\right) \\
& +\cdots+\frac{\left(t-t_{0}\right)^{m-1}}{\Gamma(1+(m-1) \alpha)} \xi^{((m-1) \alpha)}\left(t_{0}\right)
\end{aligned}
$$

Eq. (12) is equivalent to:

$$
\begin{aligned}
D_{t}^{\alpha} \xi(t)= & \frac{1}{\Gamma(m-\alpha)} \frac{d^{m}}{d t^{m}} \int_{t_{0}}^{t}(\tau-t)^{m-\alpha-1} \\
& \left(\sum_{i=0}^{m-1} \frac{1}{i !}\left(\tau-t_{0}\right)^{i} \xi^{(i)}\left(t_{0}\right)-\xi(\tau)\right) d \tau
\end{aligned}
$$

for a continuous and differentiable case. Eq. (12) is equivalent to:

$$
\begin{aligned}
D_{t}^{\alpha} \xi(t)= & \frac{1}{\Gamma(m-\alpha)} \frac{d^{m}}{d t^{m}} \int_{t_{0}}^{t}(\tau-t)^{m-\alpha-1} \\
& \left(\sum_{i=0}^{m-1} \frac{\left(\tau-t_{0}\right)^{i}}{\Gamma(1+i \alpha)} \xi^{(i \alpha)}\left(t_{0}\right)-\xi(\tau)\right) d \tau
\end{aligned}
$$

for continuous and nondifferentiable cases.

Definition 7. Keeping only the first term of $\xi_{0}(\tau)$, we give another definition of a fractional derivative in the form [23]:

$D_{t}^{\alpha} \xi(t)=\frac{1}{\Gamma(m-\alpha)} \frac{d^{m}}{d t^{m}} \int_{t_{0}}^{t}(\tau-t)^{m-\alpha-1}\left(\xi\left(t_{0}\right)-\xi(\tau)\right) d \tau$.
Hereby, $\xi$ can be continuous and possibly not differentiable anywhere.

Definition 8. We can define another fractional derivative in the form [23]:

$$
D_{t}^{\alpha} \xi(t)=\frac{1}{\Gamma(m-\alpha)} \frac{d^{m}}{d t^{m}} \int_{t_{0}}^{t}(\tau-t)^{m-\alpha-1} \xi(\tau) d \tau
$$

The above definitions of a fractional derivative are introduced by the variational iteration method.

Proposition 1. The Laplace transform of the Caputo fractional derivative is given in the form:

$$
\mathcal{L}\left\{D_{* t}^{\alpha} \xi(x, t)\right\}=s^{\alpha} \tilde{\xi}(x, s)-\sum_{k=0}^{n-1} s^{\alpha-k-1} \xi^{(k)}(x, 0),
$$

where $\tilde{\xi}(x, s)$ denote the Laplace transform of $\xi(x, t)$.

Proposition 2. The Fourier transform of the Caputo fractional derivative is given in the form:

$$
\mathcal{F}\left\{D_{* x}^{\alpha} \xi(x, t)\right\}=(i \omega)^{\alpha} \hat{\xi}(\omega, t),
$$

where $\hat{\xi}(\omega, t)$ denote the Fourier transform of $\xi(x, t)$.

\subsection{Physical understanding of the fractional derivative}

Prof. He [24] showed that fractional differential equations can best describe discontinuous media, and the fractional order is equivalent to its fractional dimensions. Now, consider a plane with a fractal structure. The shortest path between two points, $A$ and $B$, is not a line and we have:

$$
d s_{E}=k d s^{\alpha},
$$

where $d s_{E}$ is the actual distance between two terminal points, $A$ and $B, d s$ is the line distance between two points, $\alpha$ is the fractal dimension, and $k$ is a constant. Projection of the $d s_{E}$ into the horizontal direction yields Cantor-like sets, and its length can be expressed as:

$$
\Delta_{x} A B=k_{x} d x^{\alpha_{x}}
$$

where $\alpha_{x}$ are the fractal dimensions of the Cantor-like sets in the horizontal direction, and $k_{x}$ is a constant. Eq. (21) means the following transform:

$$
s_{E}=k s^{\alpha} \text {. }
$$

Inspired by this concept of a fractional derivative, we assume that the solution of the fractional differential equation can be expressed in terms of $E_{\alpha}\left(t^{\alpha}\right)$, and the Mittag-Leffler function plays a fundamental role in our study of fractional equations. 


\section{The method in action}

Now, we consider Eqs. (1) and (2). By using the properties of the homotopy perturbation method $[11,13$, $14,16,19]$, we have the following equation:

$$
\begin{aligned}
& (1-p)\left(D_{* t}^{\eta} \xi(x, t)+D_{* t}^{\gamma} \xi(x, t)+a D_{* t}^{\delta} \xi(x, t)\right) \\
& \quad+p\left(D_{* t}^{\eta} \xi(x, t)+D_{* x}^{\gamma} \xi(x, t)+a D_{* x}^{\delta} \xi(x, t)\right. \\
& \left.\quad+b \xi(x, t)+\xi^{n}(x, t)\right)=0,
\end{aligned}
$$

where $p \in[0,1]$. Now, we construct the following sequence:

$$
\xi(x, t)=\xi_{0}(x, t)+p \xi_{1}(x, t)+p^{2} \xi_{2}(x, t)+\cdots .
$$

Consequently, we will have:

$$
\begin{aligned}
& p^{0}: D_{* t}^{\eta} \xi_{0}(x, t)+D_{* x}^{\gamma} \xi_{0}(x, t)+a D_{* x}^{\delta} \xi_{0}(x, t)=0, \\
& p^{i}: D_{* t}^{\eta} \xi_{1}(x, t)+D_{* x}^{\gamma} \xi_{1}(x, t)+a D_{* x}^{\delta} \xi_{1}(x, t) \\
& \quad=-b \xi_{i-1}-\xi_{i-1}^{n}, \\
& i=1,2, \cdots,
\end{aligned}
$$

Now, in order to find a closed form representation of the solution in terms of $\xi_{i}(x, t)$, we use the method of joint Fourier-Laplace transforms. In the other words, if we apply the Fourier transform with respect to space variable $x$, then, Eq. (26) transforms into the form:

$$
D_{* t}^{\eta} \hat{\xi}_{0}(\omega, t)+(i \omega)^{\gamma} \hat{\xi}_{0}(\omega, t)+a(i \omega)^{\delta} \hat{\xi}_{0}(\omega, t)=0,
$$

where $\omega$ is the parameter of Fourier transform. Furthermore, taking the Laplace transform of Eq. (28), one will set:

$$
\begin{aligned}
s^{\eta} \tilde{\hat{\xi}}_{0}(\omega, s) & -s^{\eta-1} \hat{\xi}_{0}(\omega, 0)-s^{\eta-2} \frac{\partial \hat{\xi}_{0}(\omega, 0)}{\partial t} \\
& +(i \omega)^{\gamma} \tilde{\hat{\xi}}_{0}(\omega, s)+a(i \omega)^{\delta} \tilde{\hat{\xi}}_{0}(\omega, s)=0
\end{aligned}
$$

where $s$ is the parameter of the Laplace transform. Consequently, we obtain:

$$
\begin{aligned}
\tilde{\hat{\xi}}_{0}(\omega, s)= & \frac{s^{\eta-1}}{s^{\eta}+(i \omega)^{\gamma}+a(i \omega)^{\delta}} \hat{\xi}_{0}(\omega, 0) \\
& +\frac{s^{\eta-2}}{s^{\eta}+(i \omega)^{\gamma}+a(i \omega)^{\delta}} \frac{\partial \hat{\xi}_{0}(\omega, 0)}{\partial t} .
\end{aligned}
$$

Consequently one will set:

$$
t^{\beta-1} E_{\alpha, \beta}\left(c t^{\alpha}\right) \stackrel{\mathcal{L}}{\leftrightarrow} \frac{s^{\alpha-\beta}}{s^{\alpha}-c},
$$

it is seen that:

$$
\begin{gathered}
\hat{\xi}_{0}(\omega, t)=E_{\eta, 1}\left(-\left((i \omega)^{\gamma}+a(i \omega)^{\delta}\right) t^{\eta}\right) \hat{\xi}_{0}(\omega, 0) \\
+t E_{\eta, 2}\left(-\left((i \omega)^{\gamma}+a(i \omega)^{\delta}\right) t^{\eta}\right) \frac{\partial \hat{\xi}_{0}(\omega, 0)}{\partial t} .
\end{gathered}
$$

We can rewrite Eq. (32) in the following form:

$$
\hat{\xi}_{0}(\omega, t)=\hat{G}_{1}(\omega, t) \hat{\xi}_{0}(\omega, 0)+\hat{G}_{2}(\omega, t) \frac{\partial \hat{\xi}_{0}(\omega, 0)}{\partial t},
$$

where:

$$
\hat{G}_{1}(\omega, t)=E_{\eta, 1}\left(-\left((i \omega)^{\gamma}+a(i \omega)^{\delta}\right) t^{\eta}\right),
$$

and:

$$
\hat{G}_{2}(\omega, t)=t E_{\eta, 2}\left(-\left((i \omega)^{\gamma}+a(i \omega)^{\delta}\right) t^{\eta}\right) .
$$

Taking the inverse Fourier transform of Eq. (33), and noticing the convolution theorem of the Fourier transform, we find that:

$$
\begin{aligned}
\xi_{0}(x, t)= & \int_{-\infty}^{\infty} G_{1}(x-y, t) \xi_{0}(y, 0) d y \\
& +\int_{-\infty}^{\infty} G_{2}(x-y, t) \frac{\partial \xi_{0}(y, 0)}{\partial t} d y
\end{aligned}
$$

where:

$$
G_{1}(x, t)=\frac{1}{2 \pi} \int_{-\infty}^{\infty} E_{\eta, 1}\left(-\left((i \omega)^{\gamma}+a(i \omega)^{\delta}\right) t^{\eta}\right) e^{-i \omega x} d \omega
$$

and:

$$
G_{2}(x, t)=\frac{t}{2 \pi} \int_{-\infty}^{\infty} E_{\eta, 2}\left(-\left((i \omega)^{\gamma}+a(i \omega)^{\delta}\right) t^{\eta}\right) e^{-i \omega x} d \omega
$$

Now, applying the Fourier transform into Eq. (27), we have:

$$
\begin{aligned}
D_{* t}^{\eta} \hat{\xi}_{1}(\omega, t) & +(i \omega)^{\gamma} \hat{\xi}_{1}(\omega, t)+a(i \omega)^{\delta} \hat{\xi}_{1}(\omega, t) \\
& =-b \hat{\xi}_{0}(\omega, t)-\hat{\xi}_{0}^{2}(\omega, t) .
\end{aligned}
$$

Taking the Laplace transform of Eq. (39), we find that:

$$
\begin{aligned}
s^{\eta} \tilde{\hat{\xi}}_{1}(\omega, s) & -s^{\eta-1} \hat{\xi}_{1}(\omega, 0)-s^{\eta-2} \frac{\partial \hat{\xi}_{1}(\omega, 0)}{\partial t} \\
& +(i \omega)^{\gamma} \tilde{\hat{\xi}}_{1}(\omega, s)+a(i \omega)^{\delta} \tilde{\hat{\xi}}_{1}(\omega, s) \\
& =-b \tilde{\hat{\xi}}_{0}(\omega, s)-\tilde{\hat{\xi}}_{0}^{2}(\omega, s),
\end{aligned}
$$

consequently: 


$$
\begin{aligned}
\tilde{\hat{\xi}}_{1}(\omega, s)= & \frac{s^{\eta-1}}{s^{\eta}+(i \omega)^{\gamma}+a(i \omega)^{\delta}} \hat{\xi}_{1}(\omega, 0) \\
& +\frac{s^{\eta-2}}{s^{\eta}+(i \omega)^{\gamma}+a(i \omega)^{\delta}} \frac{\partial \hat{\xi}_{1}(\omega, 0)}{\partial t} \\
& -\frac{b}{s^{\eta}+(i \omega)^{\gamma}+a(i \omega)^{\delta}} \tilde{\hat{\xi}}_{0}(\omega, s) \\
& -\frac{1}{s^{\eta}+(i \omega)^{\gamma}+a(i \omega)^{\delta}} \tilde{\hat{\xi}}_{0}^{2}(\omega, s) .
\end{aligned}
$$

Inverting the Laplace transform with the help of the result of Relation (31), the following is obtained:

$$
\begin{aligned}
\hat{\xi}_{1}(\omega, t)= & E_{\eta, 1}\left(-\left((i \omega)^{\gamma}+a(i \omega)^{\delta}\right) t^{\eta}\right) \hat{\xi}_{1}(\omega, 0) \\
& +t E_{\eta, 2}\left(-\left((i \omega)^{\gamma}+a(i \omega)^{\delta}\right) t^{\eta}\right) \frac{\partial \hat{\xi}_{1}(\omega, 0)}{\partial t} \\
& -b \int_{0}^{t}(t-\tau)^{\eta-1} E_{\eta, \eta}\left(-\left((i \omega)^{\gamma}\right.\right. \\
& \left.\left.+a(i \omega)^{\delta}\right)(t-\tau)^{\eta}\right) \hat{\xi}_{0}(\omega, \tau) d \tau \\
& -\int_{0}^{t}(t-\tau)^{\eta-1} E_{\eta, \eta}\left(-\left((i \omega)^{\gamma}\right.\right. \\
& \left.\left.+a(i \omega)^{\delta}\right)(t-\tau)^{\eta}\right) \hat{\xi}_{0}^{2}(\omega, \tau) d \tau .
\end{aligned}
$$

Eq. (42) can be rewritten in the following form:

$$
\begin{aligned}
\hat{\xi}_{1}(\omega, t)= & \hat{G}_{1}(\omega, t) \hat{\xi}_{1}(\omega, 0)+\hat{G}_{2}(\omega, t) \frac{\partial \hat{\xi}_{1}(\omega, 0)}{\partial t} \\
& -b \int_{0}^{t}(t-\tau)^{\eta-1} \hat{G}_{3}(\omega, t-\tau) \hat{\xi}_{0}(\omega, \tau) d \tau \\
& -\int_{0}^{t}(t-\tau)^{\eta-1} \hat{G}_{3}(\omega, t-\tau) \hat{\xi}_{0}^{2}(\omega, \tau) d \tau
\end{aligned}
$$

where:

$$
\hat{G}_{3}(\omega, t)=E_{\eta, \eta}\left(-\left((i \omega)^{\gamma}+a(i \omega)^{\delta}\right) t^{\eta}\right) .
$$

Finally, the inverse Fourier transform gives the desired solution, $\xi_{1}(x, t)$, in the following form:

$$
\begin{aligned}
& \xi_{1}(x, t)=\int_{-\infty}^{\infty} G_{1}(x-y, t) \xi_{1}(y, 0) d y \\
& +\int_{-\infty}^{\infty} G_{2}(x-y, t) \frac{\partial \xi_{1}(y, 0)}{\partial t} d y \\
& -b \int_{-\infty}^{\infty} \int_{0}^{t}(t-\tau)^{\eta-1} G_{3}(x-y, t-\tau) \xi_{0}(y, \tau) d \tau d y
\end{aligned}
$$

$$
-\int_{-\infty}^{\infty} \int_{0}^{t}(t-\tau)^{\eta-1} G_{3}(x-y, t-\tau) \xi_{0}^{2}(y, \tau) d \tau d y
$$

where:

$$
G_{3}(x, t)=\frac{1}{2 \pi} \int_{-\infty}^{\infty} E_{\eta, \eta}\left(-\left((i \omega)^{\gamma}+a(i \omega)^{\delta}\right) t^{\eta}\right) e^{-i \omega x} d \omega
$$

In the same way, we can obtain $\xi_{i}(x, t)$ for $i=2,3, \cdots$, and substitute them into:

$$
\xi(x, t)=\sum_{k=0}^{\infty} \xi_{k}(x, t)
$$

to get an analytical solution for the fractional elastic beam equation.

\section{Existence and uniqueness of solution}

Theorem 1. If $f(t) \in L_{1}(0, T<\infty)$, then, the equations:

$$
\begin{aligned}
& D_{* t}^{\eta} \xi(t)=f(t), \\
& \xi(0)=c_{0}, \quad \xi^{\prime}(0)=c_{1}, \quad \xi^{[\eta]-1}(0)=c_{[\eta]-1},
\end{aligned}
$$

have a unique solution, $\xi(t) \in L_{1}(0, T)$, which satisfies the initial conditions (49).

Proof. We construct the following homotopy:

$$
D_{* t}^{\eta} \xi(t)+p(-f(t))=0 .
$$

Expanding $\xi(t)$ by use of the homotopy parameter $p$, substituting into Eq. (50) and equating the terms with the identical power of $p$, we have:

$$
\begin{aligned}
& D_{* t}^{\eta} \xi_{j}(t)=0, \quad j=0,2,3, \cdots, \\
& D_{* t}^{\eta} \xi_{1}(t)=f(t),
\end{aligned}
$$

from Eq. (51), we obtain:

$$
\xi_{j}(t)=\sum_{k=0}^{[\eta]-1} \frac{d_{k}}{k !} t^{k}, \quad j=0,2,3, \cdots .
$$

Let us construct a solution of Eq. (52). Application of the Laplace transform on Eq. (52) gives:

$$
s^{\eta} \tilde{\xi}_{1}(s)-\sum_{k=0}^{[\eta]-1} s^{\eta-k-1} \xi_{1}^{(k)}(0)=F(s),
$$

where $\tilde{\xi}_{1}(s)$ and $F(s)$ denote the Laplace transform of 
$\xi_{1}(t)$ and $f(t)$. Then, we can write:

$$
\begin{aligned}
\tilde{\xi}_{1}(s)= & s^{-\eta} F(s)+\sum_{k=0}^{[\eta]-1} s^{-k-1} \xi_{1}^{(k)}(0) \\
& =\frac{1}{s^{\eta}} F(s)+\sum_{k=0}^{[\eta]-1} \frac{\xi_{1}^{(k)}(0)}{s^{k+1}}
\end{aligned}
$$

By noticing the following Laplace transform pair [22]:

$$
t^{k} \stackrel{\mathcal{L}}{\leftrightarrow} \frac{\Gamma(k+1)}{s^{k+1}},
$$

and applying the inverse Laplace transform, we have:

$$
\xi_{1}(t)=\frac{1}{\Gamma(\eta)} \int_{0}^{t}(t-\tau)^{\eta-1} f(\tau) d \tau+\sum_{k=0}^{[\eta]-1} \frac{\xi_{1}^{(k)}(0)}{\Gamma(k+1)} t^{k}
$$

Using the rule for the Caputo fractional differentiation of the power function, we easily obtain that:

$$
D_{* t}^{\eta}\left(\frac{t^{k}}{\Gamma(k+1)}\right)=0, \quad \eta>k .
$$

It follows from Eq. (57) that $\xi_{1}(t) \in L_{1}(0, T)$. Using Eq. (58), by direct substitution of the function defined by Expression (57) in Eq. (48), we have:

$$
D_{* t}^{\eta}\left(\frac{1}{\Gamma(\eta)} \int_{0}^{t}(t-\tau)^{\eta-1} f(\tau) d \tau\right)=D_{* t}^{\eta} I_{t}^{\eta} f(t)=f(t)
$$

Therefore, $\xi_{1}(t)$ satisfies Eqs. (48) and (49) and the existence of the solution is proved.

The uniqueness follows from the linearity of fractional differentiation and the properties of the Laplace transform. Indeed, if there exist two solutions, $\xi_{1}(t)$ and $\hat{\xi}_{1}(t)$, then $\zeta(t)=\xi_{1}(t)-\hat{\xi}_{1}(t)$ must satisfy $D_{* t}^{\eta} \zeta(t)=0$. Then, the Laplace transform of $\zeta(t)$ is $\zeta(s)=0$, and, therefore $\zeta(t)=0$ almost everywhere in the considered interval, which proves that the solution in $L_{1}(0, T)$ is unique.

Theorem 2. Suppose that the homotopy perturbation method satisfies Conditions (24) and (25). Then, $E q$. (1) has a unique solution, $\xi(x, t) \in L_{1}(0, X) \times$ $(0, T)$.

Proof. Using Theorem 1, we need only prove that, the following expression, $i=1,2, \cdots$, achieved from the homotopy perturbation method on the fractional elastic beam equation has a unique solution:

$$
\begin{aligned}
D_{* t}^{\eta} \xi_{i}(x, t) & +D_{* x}^{\gamma} \xi_{i}(x, t)+a D_{* x}^{\delta} \xi_{i}(x, t) \\
& =-b \xi_{i-1}(x, t)-\xi_{i-1}^{n}(x, t) .
\end{aligned}
$$

Let us assume that $\phi(x, t) \in L_{1}(0, X) \times(0, T)$, such that:

$$
D_{* t}^{\eta} \xi_{i}(x, t)=\phi(x, t) .
$$

Using Theorem 1, we can write:

$$
\xi_{i}(x, t)=\frac{1}{\Gamma(\eta)} \int_{0}^{t}(t-\tau)^{\eta-1} \phi(x, \tau) d \tau+k_{1} t^{\eta-1}+k_{2}
$$

Substituting Eq. (62) in Eq. (60), we obtain:

$$
\phi(x, t)+D_{* x}^{\gamma} I_{t}^{\eta} \phi(x, t)+a D_{* x}^{\delta} I_{t}^{\eta} \phi(x, t)=g(x, t),
$$

where:

$$
\begin{aligned}
g(x, t)= & -b \xi_{i-1}(x, t)-\xi_{i-1}^{n}(x, t)-D_{* x}^{\gamma}\left(k_{1} t^{\eta-1}+k_{2}\right) \\
& -a D_{* x}^{\delta}\left(k_{1} t^{\eta-1}+k_{2}\right) .
\end{aligned}
$$

Hence, from Eq. (63), one will set:

$$
\begin{aligned}
& \phi(x, t)+\frac{1}{\Gamma(m-\gamma)} \int_{0}^{x} \frac{\partial^{m}}{\partial \xi^{m}} \\
& \left(\frac{1}{\Gamma(\eta)} \int_{0}^{t}(t-\tau)^{\eta-1} \phi(\xi, \tau) d \tau\right)(x-\xi)^{m-\gamma-1} d \xi \\
& +\frac{a}{\Gamma(n-\delta)} \int_{0}^{x} \frac{\partial^{n}}{\partial \xi^{n}} \\
& \quad\left(\frac{1}{\Gamma(\eta)} \int_{0}^{t}(t-\tau)^{\eta-1} \phi(\xi, \tau) d \tau\right)(x-\xi)^{n-\delta-1} d \xi \\
& =g(x, t) .
\end{aligned}
$$

Therefore, we obtain the Volterra integral equation of second kind for the function $\phi(x, t)$

$$
\phi(x, t)+\int_{0}^{x} \int_{0}^{t} K(x, \xi) K(t, \tau) \phi(\xi, \tau) d \tau d \xi=(x, t) .
$$

Then, kernel $K(x, \xi)$ can be written in the form of a weakly singular kernel:

$$
K(x, \xi)=\frac{K^{*}(x, \xi)}{(x-\xi)^{1-\mu}},
$$

where $K^{*}(x, \xi)$ is continuous and:

$$
\mu=\min (m-\gamma, n-\delta)
$$


Similarly, $g(x, t)$ can be written in the form:

$$
g(x, t)=\frac{g^{*}(x, t)}{t^{1-\nu}}
$$

where $g^{*}(x, t)$ is continuous and:

$$
\nu=\min (\eta-\gamma, \eta-\delta) \text {. }
$$

It is known that Eq. (66) with the weakly singular kernel and the right-hand side $g(x, t) \in L_{1}(0, X) \times$ $(0, T)$ has a unique solution, $\phi(x, t) \in L_{1}(0, X) \times$ $(0, T)$. Then, according to Theorem 1 , the unique solution, $\xi_{i}(x, t) \in L_{1}(0, X) \times(0, T)$, of Eq. (61) can be determined using Eq. (62). This ends the proof of Theorem 2.

\section{Convergence analysis}

Theorem 3. Suppose that the homotopy perturbation method satisfies Conditions (24) and (25). Then, our proposed scheme to approximate the solution of Eq. (1) is convergent.

Proof. Consider a fractional elastic beam equation in the form of Eq. (1). If we use the homotopy perturbation method, from Eqs. (32) and (43), we have:

$$
\begin{aligned}
\left|\hat{\xi}_{0}(\omega, t)\right| & \leq\left|\hat{\xi}_{0}(\omega, 0)\right| E_{\eta, 1}\left(-\left(\left(i \omega^{\gamma}+a(i \omega)^{\delta}\right) t^{\eta}\right)\right. \\
& +\left|\frac{\partial \hat{\xi}_{0}(\omega, 0)}{\partial t}\right| t E_{\eta, 2}\left(-\left((i \omega)^{\gamma}+a(i \omega)^{\delta}\right) t^{\eta}\right),
\end{aligned}
$$

and:

$$
\begin{aligned}
\left|\hat{\xi}_{m}(\omega, t)\right| \leq & \left|\hat{\xi}_{m}(\omega, 0)\right| E_{\eta, 1}\left(-\left((i \omega)^{\gamma}+a(i \omega)^{\delta}\right) t^{\eta}\right) \\
& +\left|\frac{\partial \hat{\xi}_{m}(\omega, 0)}{\partial t}\right| t E_{\eta, 2}\left(-\left((i \omega)^{\gamma}+a(i \omega)^{\delta}\right) t^{\eta}\right) \\
& +b \int_{0}^{t}\left|\hat{\xi}_{m-1}(\omega, \tau)\right|(t-\tau)^{\eta-1} E_{\eta, \eta}\left(-(i \omega)^{\gamma}\right. \\
& \left.\left.+a(i \omega)^{\delta}\right)(t-\tau)^{\eta}\right) d \tau \\
& +\int_{0}^{t}\left|\hat{\xi}_{m-1}^{2}(\omega, \tau)\right|(t-\tau)^{\eta-1} E_{\eta, \eta}\left(-(i \omega)^{\gamma}\right. \\
& \left.\left.+a(i \omega)^{\delta}\right)(t-\tau)^{\eta}\right) d \tau, \\
m=1,2, & \ldots,
\end{aligned}
$$

and, by noticing the following properties of the Mittag-
Leffler function:

$$
\begin{aligned}
& \int_{0}^{t}(t-\tau)^{\alpha-1} E_{\alpha, \alpha}\left((t-\tau)^{\alpha}\right) d \tau=t^{\alpha} E_{\alpha, \alpha+1}\left(t^{\alpha}\right), \\
& E_{\alpha, \alpha+1}\left(t^{\alpha}\right)=\frac{1}{t^{\alpha}}\left(E_{\alpha, 1}\left(t^{\alpha}\right)-1,\right.
\end{aligned}
$$

we obtain:

$$
\begin{aligned}
& \sum_{k=0}^{\infty} \hat{\xi}_{k}(\omega, t) \leq \lambda_{1} E_{\eta, 1}\left(-\left((i \omega)^{\gamma}+a(i \omega)^{\delta}\right) t^{\eta}\right) \\
& \quad+\lambda_{2} t E_{\eta, 2}\left(-\left((i \omega)^{\gamma}+a(i \omega)^{\delta}\right) t^{\eta}\right) \\
& \quad+\lambda_{3} \int_{0}^{t}(t-\tau)^{\eta-1} E_{\eta, \eta}\left(-\left((i \omega)^{\gamma}\right.\right. \\
& \left.\left.\quad+a(i \omega)^{\delta}\right)(t-\tau)^{\eta}\right) d \tau \leq \Lambda E_{\eta, 1}\left(-\left(|\omega|^{\gamma}+a|\omega|^{\delta}\right) t^{\eta}\right.
\end{aligned}
$$

Consequently, inverting the Fourier transform, by means of the following Fourier transform pair [21]:

$$
\begin{aligned}
& \frac{t^{\beta-1}}{\sqrt{\pi}|x|} H_{2,3}^{2,1}\left(\left.\frac{|x|^{\gamma}}{2^{\gamma} c t^{\alpha}}\right|_{(1,1),\left(\frac{1}{2}, \frac{\gamma}{2}\right),\left(1, \frac{\gamma}{2}\right)} ^{(1,1),(\beta, \alpha)}\right) \\
& \quad \stackrel{\mathcal{F}}{\leftrightarrow} t^{\beta-1} E_{\alpha, \beta}\left(-c|\omega|^{\gamma} t^{\alpha}\right),
\end{aligned}
$$

and the comparison test implies that the series $\sum_{k=0}^{\infty} \xi_{k}(x, t)$ converges.

\section{Stability analysis}

Theorem 4. Under Assumptions (24) and (25), Eq. (1) has a solution $\xi(x, t)$, which is asymptotically stable.

Proof. Consider a fractional elastic beam equation in the form of Eq. (1). Assume that $|\eta-\tilde{\eta}|<\epsilon, \mid \gamma-$ $\tilde{\gamma} \mid<\epsilon$ and $|\delta-\tilde{\delta}|<\epsilon$. Let $\xi(x, t)$ and $\zeta(x, t)$ be, respectively, the solution of Eq. (1) and the following fractional elastic beam equation:

$$
D_{* t}^{\tilde{\eta}} \zeta+D_{* x}^{\tilde{\gamma}} \zeta+a D_{* x}^{\tilde{\delta}} \zeta+b \zeta+\zeta^{n}=0 .
$$

As a direct consequence of Eqs. (32) and (43), we obtain:

$$
\begin{aligned}
& |\xi(\omega, t)-\zeta(\omega, t)| \\
& =\mid \hat{\xi}_{0}(\omega, 0)\left\{\sum_{j=0}^{\infty} \frac{\left((i \omega)^{\gamma}+a(i \omega)^{\delta}\right)^{j} t^{j \tilde{\eta}}}{\Gamma(1+j \tilde{\eta})}\right. \\
& \left.\quad-\sum_{j=0}^{\infty} \frac{\left((i \omega)^{\gamma}+a(i \omega)^{\delta}\right)^{j} t^{j(\tilde{\eta}+\epsilon)}}{\Gamma(1+j(\tilde{\eta}+\epsilon))}\right\}
\end{aligned}
$$




$$
\begin{aligned}
& +t \frac{\partial \hat{\xi}_{0}(\omega, 0)}{\partial t}\left\{\sum_{j=0}^{\infty} \frac{\left((i \omega)^{\gamma}+a(i \omega)^{\delta}\right)^{j} t^{j \tilde{\eta}}}{\Gamma(2+j \tilde{\eta})}\right. \\
& \left.-\sum_{j=0}^{\infty} \frac{\left((i \omega)^{\gamma}+a(i \omega)^{\delta}\right)^{j} t^{j(\tilde{\eta}+\epsilon)}}{\Gamma(2+j(\tilde{\eta}+\epsilon))}\right\} \\
& +b \int_{0}^{t}(t-\tau)^{\eta-1} \hat{\xi}_{0}(\omega, \tau) \\
& \left\{\sum_{j=0}^{\infty} \frac{\left((i \omega)^{\gamma}+a(i \omega)^{\delta}\right)^{j}(t-\tau)^{j \tilde{\eta}}}{\Gamma(\tilde{\eta}+j \tilde{\eta})}\right. \\
& \left.-\sum_{j=0}^{\infty} \frac{\left((i \omega)^{\gamma}+a(i \omega)^{\delta}\right)^{j}(t-\tau)^{j(\tilde{\eta}+\epsilon)}}{\Gamma(\tilde{\eta}+\epsilon+j(\tilde{\eta}+\epsilon))}\right\} d \tau \\
& +\int_{0}^{t}(t-\tau)^{\eta-1} \hat{\xi}_{0}^{2}(\omega, \tau) \\
& \left\{\sum_{j=0}^{\infty} \frac{\left((i \omega)^{\gamma}+a(i \omega)^{\delta}\right)^{j}(t-\tau)^{j \tilde{\eta}}}{\Gamma(\tilde{\eta}+j \tilde{\eta})}\right. \\
& \left.-\sum_{j=0}^{\infty} \frac{\left((i \omega)^{\gamma}+a(i \omega)^{\delta}\right)^{j}(t-\tau)^{j(\tilde{\eta}+\epsilon)}}{\Gamma(\tilde{\eta}+\epsilon+j(\tilde{\eta}+\epsilon))}\right\} d \tau \mid \\
& \leq K \sum_{j=0}^{\infty} \frac{t^{j \tilde{\eta}}\left(\left((i \omega)^{\gamma}+a(i \omega)^{\delta}\right) \sum_{i=0}^{\infty}(-1)^{i} t^{i \epsilon}\right)}{\Gamma(1+j \tilde{\eta})} \\
& =K E_{\tilde{\eta}, 1}\left(-\lambda t^{\tilde{\eta}}\right), \quad K, \lambda>0 .
\end{aligned}
$$

Therefore, by our assumption on $\eta, \gamma$ and $\delta$, by means of the Fourier transform pair in Eq. (76), we find that the solution is asymptotically stable.

\section{Results and discussion}

In this section, we apply the method presented in Section 3 to solve the following two test examples. The observations are depicted through Figures 1 and 2. Figures 1 and 2 show the comparison between the obtained solutions in [25] and our approximate solutions for the nonlinear fractional beam equation, i.e. for:

$$
\begin{aligned}
& D_{* t}^{\eta_{1}} \xi(x, t)+D_{* x}^{\gamma_{1}} \xi(x, t)+a_{1} D_{* x}^{\delta_{1}} \xi(x, t)+b_{1} \xi \\
&+\xi^{2}(x, t)=0 \\
& \xi(x, 0)= \frac{1}{e^{0.1136 x}+0.5+0.0625 e^{-0.1136 x}}, \\
& \frac{\partial \xi(x, 0)}{\partial t}=\frac{0.8 e^{0.1136 x}-0.0625 \times 0.8^{-0.1136 x}}{\left(e^{0.1136 x}+0.5+0.0625 e^{-0.1136 x}\right)^{2}},
\end{aligned}
$$

and the following equation that can be achieved from

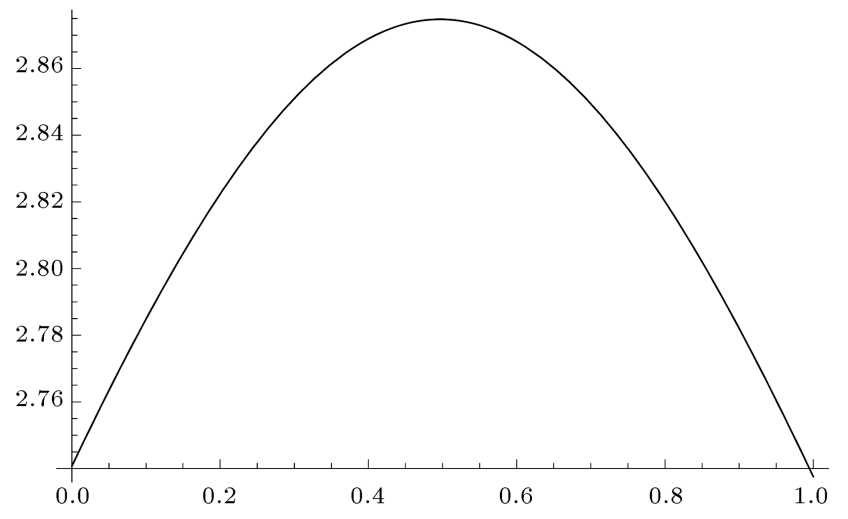

Figure 1. Approximate solution of Eq. (79) where $x, t \in[-10,10], \eta_{1}=2, \gamma_{1}=4, \delta_{1}=2, a_{1}=2$ and $b_{1}=-0.66$.

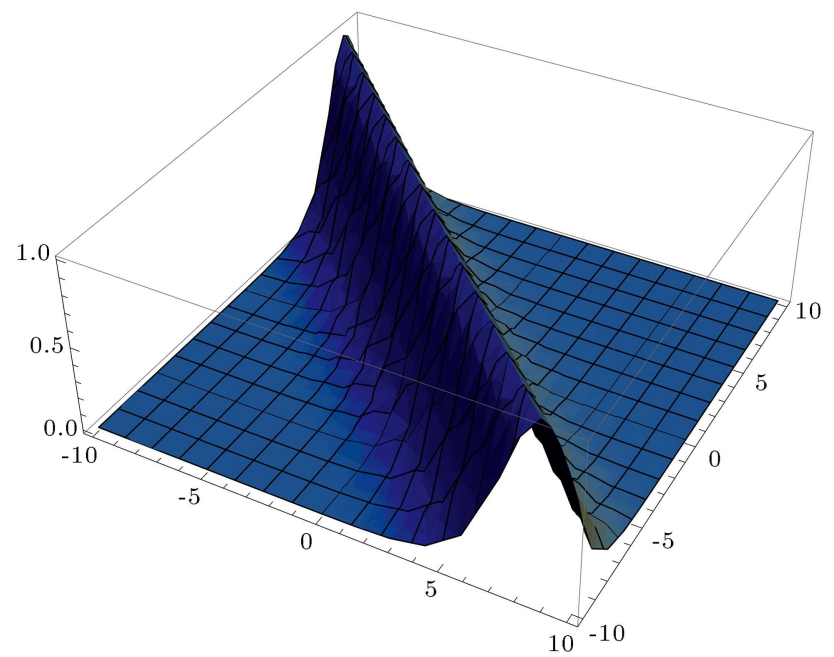

Figure 2. Approximate solution of Eq. (82) where $x \in[0,1], \alpha=1, a_{2}=-9$ and $b_{2}=-4$.

Eq. (1) using a travelling wave transformation:

$$
\begin{aligned}
& D_{* x}^{4 \alpha} \xi(x)+a_{2} D_{* x}^{2 \alpha} \xi(x)+b_{2} \xi(x)+\xi^{3}(x)=0, \\
& \xi(0)=\xi(1)=2.735, \quad \xi^{\prime}(0)=0.465, \\
& \xi^{\prime}(1)=-0.465 .
\end{aligned}
$$

We can obtain $\xi(x, t)$, as follows:

$$
\xi(x, t)=\sum_{k=0}^{\infty} \xi_{k}(x, t),
$$

where, in the first example:

$$
\begin{aligned}
\xi_{0}(x, t)= & \int_{-\infty}^{\infty} G_{1}(x-y, t) \xi(y, 0) d y \\
& +\int_{-\infty}^{\infty} G_{2}(x-y, t) \frac{\partial \xi(y, 0)}{\partial t} d y
\end{aligned}
$$

and: 


$$
\begin{aligned}
\xi_{k}(x, t) & \\
= & -b_{1} \int_{-\infty}^{\infty} \int_{0}^{t}(t-\tau)^{\eta_{1}-1} G_{3}(x-y, t-\tau) \xi_{k-1}(y, \tau) d \tau d y \\
& -\int_{-\infty}^{\infty} \int_{0}^{t}(t-\tau)^{\eta_{1}-1} G_{3}(x-y, t-\tau) \xi_{k-1}^{2}(y, \tau) d \tau d y \\
k & =1,2, \cdots,
\end{aligned}
$$

where $G_{1}(x, t), G_{2}(x, t)$ and $G_{3}(x, t)$ are defined by Eqs. (37), (38) and (46), and in the second example:

$$
\xi_{0}(x)=c_{0}+c_{1} \frac{x^{\alpha}}{\alpha !}+c_{2} E_{\alpha}\left(\sqrt{a_{2}} x^{\alpha}\right)+c_{3} E_{\alpha}\left(-\sqrt{a_{2}} x^{\alpha}\right),
$$

where $c_{i}, i=0,1,2,3$ can be determined by boundary conditions and:

$$
\begin{aligned}
\xi_{k}(x)= & -b_{2} \int_{-\infty}^{\infty} G_{3}(x-y) \xi_{k-1}(y) d y \\
& -\int_{-\infty}^{\infty} G_{3}(x-y) \xi_{k-1}^{3}(y) d y, \\
k=1,2, \cdots &
\end{aligned}
$$

where:

$$
G_{3}(x)=\frac{1}{2 \pi} \int_{-\infty}^{\infty} \frac{1}{(i \omega)^{4 \alpha}+a_{2}(i \omega)^{2 \alpha}} e^{-i \omega x} d \omega .
$$

It is clear that the obtained results aided by Mathematica 7.0 software are in close agreement with those obtained in [25]. Also, the method required to solve these problems uses only two iterations, while, in [25], the exp-function method required to solve the mentioned problems for an integer order of derivatives requires many manipulations. Therefore, for this case study, our proposed scheme obtained results having greater precision, a wider range, and require less iteration than the exp-function method.

\section{Conclusions}

In this manuscript, the homotopy perturbation method, with the help of joint Fourier-Laplace transforms, has been successfully applied to compute an analytical solution of a nonlinear fractional beam equation. The convergence and stability of the homotopy perturbation method solution, as applied to this equation, have been thoroughly investigated. The obtained results show that our approach provides the solution, in terms of the convergent series, with easily computable components in a direct way, and is very effective and convenient for dealing with nonlinear fractional beam equations. Finally, the scheme can be applied to many physical problems, e.g., irregular signals, fractional Brownian motion and scale relativity.

\section{References}

1. Baleanu, D., Diethelm, K., Scalas, E. and Trujillo, J.J., Fractional Calculus Models and Numerical Methods, World Scientific (2012).

2. He, J.H. "Some applications of nonlinear fractional differential equations and their applications", Bull. Sci. Technol., 15(2), pp. 86-90 (1999).

3. Agrawal, O.P. "A general solution for a fourth-order fractional diffusion-wave equation defined in a bounded domain", Comput. Struct., 79, pp. 1497-1501 (2001).

4. He, J.H. "Homotopy perturbation technique", Comput. Methdos Appl. Mech. Engrg., 178, pp. 257-262 (1999).

5. Odibat, Z. and Momani, S. "Modified homotopy perturbation method: Application to quadratic Riccati differential equation of fractional order", Chaos, Solitons Fractals, 36, pp. 167-174 (2008).

6. Dehghan, M. "A new operational matrix for solving fractional-order differential equations", Comput. Math. Appl., 59, pp. 1326-1336 (2010).

7. Kumar, S. "A new analytical modelling for telegraph equation via Laplace transform", Appl. Math. Modell., 38(13), pp. 3154-3163 (2014).

8. Kumar, S. "A numerical study for solution of time fractional nonlinear Shallow-water equation in oceans", Zeits. Naturfor. A, 68a, pp. 1-7 (2013).

9. Kumar, S. "Numerical computation of time-fractional Fokker-Planck equation arising in solid state physics and circuit theory", Zeits. Naturfor., 68a, pp. 1-8 (2013).

10. Kumar, S. and Rashidi, M.M. "New analytical method for gas dynamics equation arising in shock fronts", Comput. Phys. Commun., 185(7), pp. 1947-1954 (2014).

11. He, J.H. "Homotopy perturbation method: a new nonlinear analytical technique", Appl. Math. Comput., 135, pp. 73-79 (2003).

12. He, J.H. "A tutorial review on fractal spacetime and fractional calculus", International Journal of Theor. Phys., 53(11), pp. 3698-3718 (2014).

13. Jafari, H. and Momani, S. "Solving fractional diffusion and wave equations by modified homotopy perturbation method", Phys. Lett. A, 370, pp. 388-396 (2007).

14. Dehghan, M. and Shakeri, F. "The numerical solution of the second Painleve equation", Numer. Meth. Part. D.E., 25(5), pp. 1238-1259 (2009).

15. Golmankhaneh, A.R.K., Golmankhaneh, A.K. and Baleanu, D. "Homotopy perturbation method for solv- 
ing a system of Schordeinger-KdV equation", Rom. Journ. Phys., 63(3), pp. 609-623 (2011).

16. Gepreel, K.A. and Omran, S. "The exact solutions for the nonlinear partial fractional differential equations", Chinese Phys., 21, pp. 110204-110211 (2012).

17. Herzallah, M.A.E. and Gepreel, K.A. "Approximate solution to the time-space fractional cubic nonlinear Schrodinger equation", Appl. Math. Modell., 36(11), pp. 5678-5685 (2012).

18. Gepreel, K.A. "The homotopy perturbation method to the nonlinear fractional Kolmogorov-PetrovskiiPiskunov equations", Appl. Math. Lett., 24, pp. 14281434 (2011).

19. Sayevand, K. "Efficient solution of fractional initial value problems using expanding perturbation approach", J. Math. Comput. Sci., 8(4), pp. 359-366 (2014).

20. Rektorys, K., Handbook of Applied Mathematics, 1(11), SNTL, Prague (1988).

21. Yu, R. and Zhang, H. "New function of Mittag-Leffler type and its application in the fractional diffusion-wave equation", Chaos Solit. Fract., 30, pp. 946-955 (2006).

22. Podlubny, I., Fractional Differential Equations, San Diego, Academic Press (1999).

23. He, J.H., Elagan, S.K. and Li, Z.B. "Geometrical explanation of the fractional complex transform and derivative chain rule for fractional calculus", Phys. Lett. A, 376(4), pp. 257-259 (2012).

24. He, J.H. "String theory in a scale dependent discontinuous space-time", Chaos Solit. Fract., 36(3), pp. 542545 (2008).

25. Zahra, K.A.A. and Hussain, M.A.A. "Exp-function method for solving nonlinear beam equation", Int. Math. Forum., 6, pp. 2349-2359 (2011).

\section{Biographies}

Khosro Sayevand received his BS degree from the University of Mazandaran, Iran, in 1997, his MS degree from Amirkabir University of Technology, Tehran, Iran, in 2000, and his PhD degree from Iran University of Science and Technology, Iran, in 2010, all in Applied Mathematics. He is currently Associate Professor at Malayer University, Iran. His current research interest mainly covers fractional calculus and numerical analysis. He has published more than 40 articles in these areas in ISI-listed journals.

Kazem Pichaghchi received BS and MS degrees in Mathematics from Semnan University, Iran, and Azarbaijan University of Tarbiat Moallem, Iran, in 2009 and 2011, respectively. His research interests include numerical analysis. At present he is a $\mathrm{PhD}$ degree student at Malayer University, Iran. 